\title{
Impact Induced Delamination Detection and Quantification with Guided Wavefield Analysis
}

\author{
Zhenhua Tian ${ }^{1}$, Cara A.C. Leckey ${ }^{2}$, Lingyu Yu ${ }^{1 *}$, Jeffrey P. Seebo ${ }^{3}$ \\ ${ }^{1}$ Department of Mechanical Engineering, University of South Carolina, Columbia, SC \\ ${ }^{2}$ Nondestructive Evaluation Sciences Branch, NASA Langley Research Center, Hampton, VA \\ ${ }^{3}$ Analytical Mechanics Associates, Inc., Hampton, VA
}

\begin{abstract}
This paper studies impact induced delamination detection and quantification methods via guided wavefield data and spatial wavenumber imaging. In this study, the complex geometry impact-like delamination damage in a composite laminate is created through the quasi-static indention technique. To detect and quantify the delamination damage, the guided ultrasonic waves are excited through a piezoelectric actuator, and the guided wavefields are measured by a scanning laser Doppler vibrometer. The acquired guided wavefield data represent wave propagation in the composite plate and includes complex wave interaction at the delamination region. To process the wavefield data and evaluate the delamination damage, the measured wavefields are analyzed through the spatial wavenumber imaging method which can generate an image containing the dominant local wavenumber at each spatial location. For a proof of concept, the approach is first applied to a single Teflon insert simulating a delamination, and then to the complex geometry impactlike delamination damage. The results show that the spatial wavenumber imaging can not only determine the delamination location, but also provide quantitative information regarding the delamination size and shape. The detection results for the impact induced delamination are compared to an ultrasonic C-scan image and wavenumber images are studied for two different excitation frequencies. Fairly good agreement is observed for portions of the delamination, and differences in wavenumber are observed at the two different frequencies. Results demonstrate that the spatial wavenumber imaging is a promising technique for yielding delamination location and size information.
\end{abstract}

Keywords: Guided waves, Composites, Delamination, Frequency-wavenumber analysis, Laser vibrometer

\section{INTRODUCTION}

Aerospace composites can incur unexpected damage due to impact events or due to stressing of the material during off-nominal loading events. Laminated composites are susceptible to delamination damage due to their weak transverse tensile and interlaminar shear strengths (as compared to their in-plane properties) [1]. Since delamination damage can occur internally, such that it is not readily visible on the composite surface, hidden delamination damage must be detected via nondestructive inspection and/or structural health monitoring (SHM) methods and evaluated before it becomes critical [2]. Development of reliable and quantitative techniques to detect delamination damage in laminated composites is imperative for the safe operation and optimal design of next-generation composite aircrafts.

Guided ultrasonic waves have proven to be an effective and efficient method for SHM, due to their ability to cover large areas while maintaining high sensitivity to small defects [3]. Advances in guided wave based SHM technologies over the last decade have demonstrated the feasibility of detecting and locating damage in composite structural components [3-12]. Due to the complex wave interaction that occurs when hidden delamination damage is present, tremendous efforts have been put into guided wave delamination detection methodologies [2, 13-20]. Petculescu et al. used a group delay measurement technique for the detection and sizing of delaminations in unidirectional and cross-ply composites [14]. They found that the delamination affects the travel time of ultrasonic wave packets. Staszewski et al. used 3-D laser vibrometry to locate delamination damage in a composite plate and estimate damage severity, demonstrating that the delamination can be revealed by the amplitude profiles of Lamb waves [2]. Purekar and Pines demonstrated the application of piezoelectric phased sensor arrays to detect the presence of delamination damage in a

\footnotetext{
*Corresponding author. Email: yu3@cec.sc.edu
} 
composite laminate [15]. Michaels et al. used guided wavefield images and frequency-wavenumber domain analysis to study the wave interactions with structural discontinuities in composites [16]. Sohn et al. proposed a "standing wave filter" which can isolate the standing wave components of a given wavefield for delamination detection in composites [18]. Rogge and Leckey presented a local wavenumber domain analysis to process the guided wavefield and they demonstrated this method can be used to quantify the delamination depth and size [19]. Flynn et al. presented a structural imaging method by using frequency-wavenumber filtering and local wavenumber estimation, and they showed this method was capable of detecting the wall-thinning in an aluminum plate and a steel pipe, and local impact delamination in a complicated composite component[20]. More recently, Mesnil et al. presented instantaneous and local wavenumber estimations for delamination damage quantification in composites [21].

This paper studies impact induced delamination damage detection and quantification by using guided wavefield data and spatial wavenumber imaging. We experimentally acquire guided wavefield data of wave interaction at the delamination damage, and apply wavefield analysis to analyze the complex wave interaction phenomenon and to quantify the delamination damage. In the test, the guided waves are generated by surface bonded piezoelectric transducers (lead zirconate titanate, PZT) and the guided wavefields are acquired through a non-contact scanning laser Doppler vibrometer (SLDV). With this PZT-SLDV sensing, we acquire the time-space wavefield, which represents guided wave propagation in the composite laminate and includes guided wave interaction with the delamination damage. To detect and quantify the delamination damage, the measured guided wavefields are analyzed through the spatial wavenumber imaging method, which generates an image containing the dominant local wavenumber at each spatial location. This spatial wavenumber image can thus be used for delamination detection. For the proof of concept, the approach is applied to a single Teflon insert simulating a delamination at first and then to the complex geometry impactlike delamination damage. In prior work, impact damage wavenumber imaging was performed using a single frequency excitation. In this paper, the prior wavenumber result is compared to new results for a lower excitation frequency to study the dependence on frequency of the damage quantification results. The results for the Teflon case show that the spatial wavenumber imaging can not only determine the delamination location, but also provide quantitative evaluation regarding the delamination size and shape for simple damage types. For the impact damage, the results demonstrate that accurate quantification is more challenging and depends on excitation frequency.

\section{WAVE INTERACTION WITH DELAMINATION DAMAGE}

When guided waves propagate in a delaminated composite, multiple reflections within the delamination region can occur, as shown in references [18, 22-25]. Hence, a considerable amount of ultrasonic energy is "trapped" above and below the delaminated region until eventually the energy dissipates. Ramadas et al. studied the interaction of the antisymmetric $\mathrm{A}_{0}$ mode with symmetric delaminations [23]. They identified multiple reflections in the delamination region. The trapped energy phenomenon of guided wave propagation in delaminated composites has also been studied experimentally through the use of wavefield images obtained from laser vibrometry tests $[18,24]$. It is observed that upon entering the delaminated region, the incident waves will be split above and below the delamination and then propagate independently through upper and lower laminates (single delamination scenario being assumed). After that, a significant portion of the waves are reflected back from the far edge of the delamination (exit region). The reflected waves then pass back above (or below) the delamination region and experience reflections at the original entrance to the delamination region. The process is repeated until the energy is dissipated, as found in the simulation work reported by Hayashi and Kawashima[22]. Glushkov et al. also observed similar wave energy trapping and localization in a strip delamination by using laser vibrometry to record the wavefield [25]. Since the material layers above and below the delamination have different thicknesses from the original plate thickness, Tian et al found the waves have different wave propagation characteristics, such as wave speed, wave mode and wavenumber [26]. Therefore, it is expected that by analyzing the guided wave signals, the delamination dimensions can be quantified. However, due to the complex wave interaction that occurs when hidden delamination damage is present, tremendous efforts have been put into delamination detection methodologies, such as references [12-17, 19]. Prior work by Rogge and Leckey demonstrated that the wavenumber information can be used to determine the approximate depth and size of near-surface delamination damage [19]. In this paper, we demonstrate quantitative characterization of impact-induced delaminating damage utilizing the guided waves and the wavenumber information. 


\section{TEFLON INSERT INDUCED DELAMINATION DETECTION AND QUANTIFICATION}

To investigate the guided wave interaction with delamination damage and establish the delamination detection method, we started from a simple case with a square Teflon insert to simulate delamination damage. Two $380 \mathrm{~mm} \times 380$ mm 8-ply IM7/8552 composite laminate plates with layup $\left[0_{2} / 90_{2}\right]_{\mathrm{s}}$ were fabricated at NASA Langley Research Center. One plate is the pristine, while the other contains a delamination created by inserting a $20 \mathrm{~mm} \times 20 \mathrm{~mm}$ Teflon film under the second ply, as indicated in Figure 1Figure $1 \mathrm{a}$ and $\mathrm{b}$.

The hybrid PZT-laser vibrometry test was performed to excite guided waves, acquire the guided wavefields, and visualize the wave interaction with the delamination damage. Figure 1Figure 1c gives the overall experimental setup. A surface bonded $0.2 \mathrm{~mm}$ thick $7 \mathrm{~mm}$ round PZT patch is used to generate guided waves. The excitation signal is a 3-cycle toneburst at frequency $300 \mathrm{kHz}$, generated from the function generator (model: Agilent 33522B). Before the excitation signal is applied to the PZT, the excitation is amplified to $40 \mathrm{~V}$ by a power amplifier (model: Krohn-Hite 1506). A scanning laser Doppler vibrometer (model: Polytec PSV-400-M2) is used to acquire the time-space wavefield over the scan area (shown in Figure 1Figure 1a). This Polytec model is a 1D SLDV, and therefore only the motion along the laser beam can be measured. In the test, the laser beam is placed normal to the plate surface such that only the out-of-plane wave velocities are measured and analyzed. Scanning is performed in the $40 \mathrm{~mm} \times 55 \mathrm{~mm}$ scan area (shown in Figure 1Figure 1a) with a spatial resolution of $0.2 \mathrm{~mm}$. At each point the measurement is averaged 20 times to improve the signal to noise ratio.

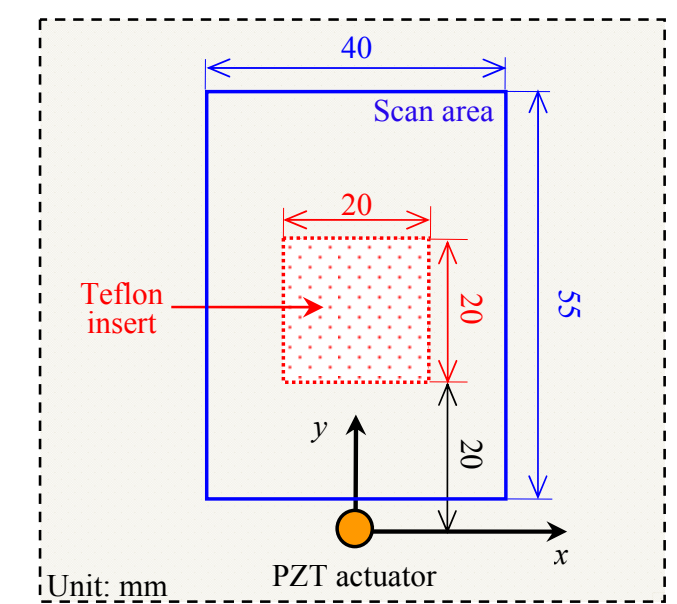

(a)

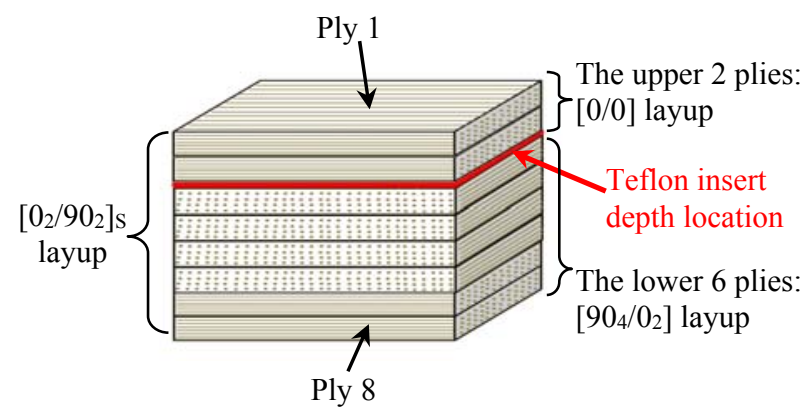

(b)

(c)

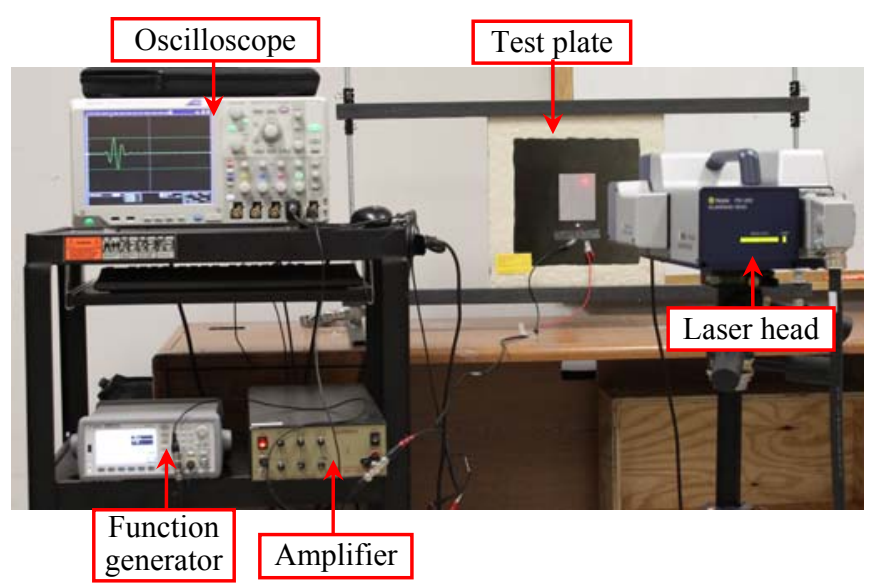

Figure 1. The scanning laser vibrometry test on a composite plate with a Teflon insert: (a) the sensing layout, (b) the side view of the $\left[0_{2} / 90_{2}\right] \mathrm{s}$ composite test plate with a Teflon insert, (c) the overall experimental setup. 
The time-space data $u(\mathbf{x}, t)$ acquired from the SLDV contains time $(t)$ signals obtained at each SLDV scan point $\mathbf{x}(x, y)$. The signals recorded at all spatial points can be presented as wavefield images at a particular propagation time. Figure 2Figure 2a shows a wavefield image for the pristine plate at $15 \mu \mathrm{s}$ after the initial excitation. Two wave modes, a faster $\mathrm{S}_{0}$ mode and a slower $\mathrm{A}_{0}$ mode, can be observed. Figure 2Figure $2 \mathrm{~b}$ shows the wavefield image for the plate containing the Teflon delamination at the same point in time. Wave interactions with the delamination are shown (delamination boundary denoted by the dotted box) after the $\mathrm{S}_{0}$ mode has passed through the damage region. Waves are observed propagating forward and backward between lower $(\mathrm{y}=20 \mathrm{~mm})$ and upper delamination $(\mathrm{y}=40 \mathrm{~mm})$ boundaries.

The guided wavefield measured from the SLDV contains abundant information regarding the wave propagation and interaction. Various methods have been used to analyze such wavefield data, such as wavefield amplitude profile, multidimensional Fourier transform, frequency-wavenumber filtering, standing wave filtering, instantaneous and local wavenumber analysis and phase gradient method[16, 18-21, 27-31]. Wavefield analysis can unveil the wave propagation and interaction details, such as the frequency-wavenumber components, wave mode information, mode conversions and damage locations. In this study, we apply the spatial wavenumber imaging method $[26,32]$ to analyze the guided wavefield and evaluate the delamination damage.

Figure 3Figure 3 shows the spatial wavenumber image calculated from the wavefield data of the Teflon delaminated plate at the excitation frequency of $300 \mathrm{kHz}$. The spatial wavenumber image indicates a region around 20 $\mathrm{mm}$ by $20 \mathrm{~mm}$ in size, which has significantly larger wavenumber values than the rest of the plate. This region matches well with the Teflon insert delamination location and size (indicated by the red dotted box in Figure 3Figure 3). Clearly the delamination affects the wavenumber of the waves that propagate through the region. The spatial wavenumber shows its potential for detecting and quantifying the delamination damage.

Table 1 Material properties

\begin{tabular}{lllllllllll}
\hline$\rho\left(\mathrm{kg} / \mathrm{m}^{3}\right)$ & $\mathrm{E}_{1}(\mathrm{GPa})$ & $\mathrm{E}_{2}(\mathrm{GPa})$ & $\mathrm{E}_{3}(\mathrm{GPa})$ & $\mathrm{G}_{12}(\mathrm{GPa})$ & $\mathrm{G}_{13}(\mathrm{GPa})$ & $\mathrm{G}_{23}(\mathrm{GPa})$ & $v_{12}$ & $v_{13}$ & $v_{23}$ & Thickness $(\mathrm{mm})$ \\
\hline 1570 & 171.4 & 9.08 & 9.08 & 5.29 & 5.29 & 2.80 & 0.32 & 0.32 & 0.5 & 0.1068 \\
\hline
\end{tabular}
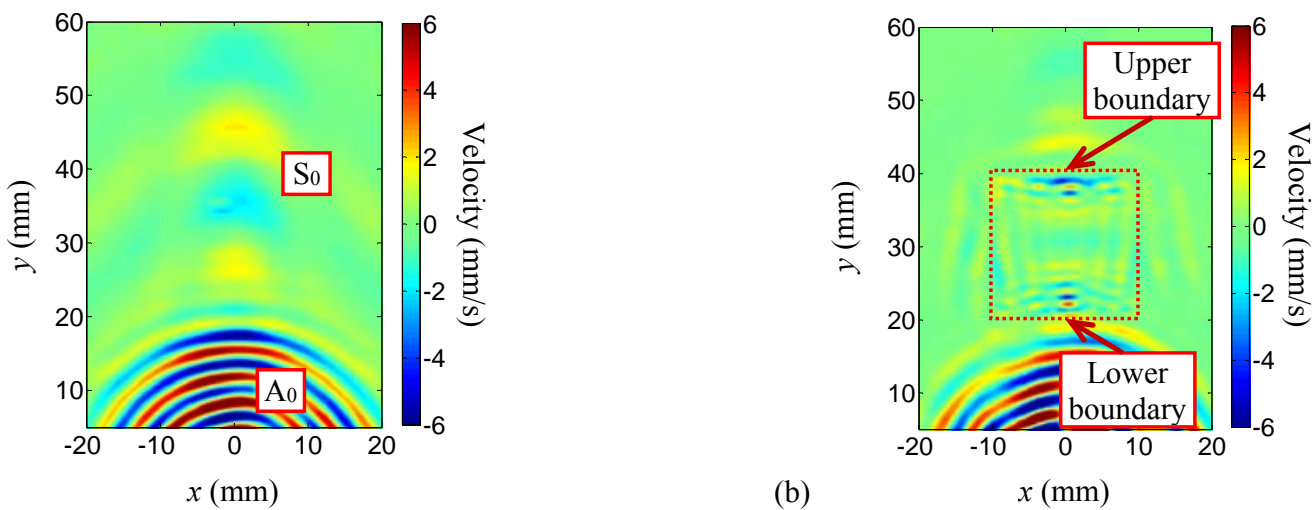

Figure 2. SLDV wavefield images at $15 \mu \mathrm{s}$ : (a) for the pristine plate, and (b) for the plate with a Teflon insert. The red dotted box indicates the actual Teflon insert. 


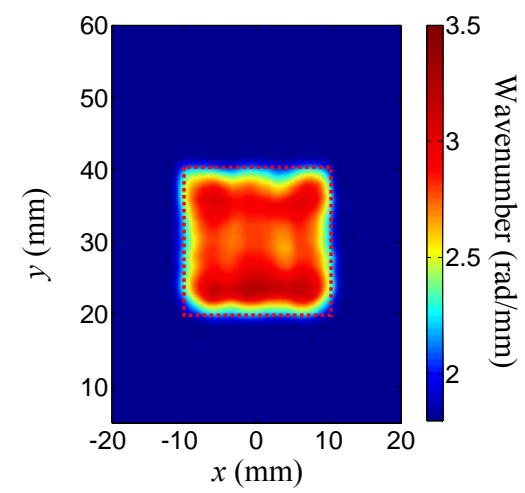

Figure 3. Spatial wavenumber image of the Teflon delaminated plate. The red dotted box indicates the actual Teflon insert.

\section{IMPACT-INDUCED DELAMINATION DETECTION AND QUANTIFICATION}

The previous section has shown that the spatial wavenumber imaging yields quantitative information of guided wave interaction in the delamination region. Moreover, the spatial wavenumber imaging detects the delamination location and size. In this section, the spatial wavenumber imaging is used to detect and quantify impact-like delamination damage in laminated composite plates. The frequency dependence of the resulting wavenumber image is also studied through comparisons of results at two different excitation frequencies $(100 \mathrm{kHz}$ and $500 \mathrm{kHz})$.

\subsection{Damage growth in a CFRP plate}

The delamination damage is created through the quasi-static indentation technique, which has been shown by previous authors to be an ideal method for controlled growth of impact-like damage in composite laminates[33]. The composite plate used for damage growth is a 26 ply IM7/8552 $381 \mathrm{~mm} \times 381 \mathrm{~mm}$ laminate with a quasi-isotropic layup of $\left[\left(0_{2} / 45_{2} /-45_{2}\right)_{2} 90\right]_{\mathrm{s}}$. The material properties of each ply are given in Table 1Table 1 . The composite plate was loaded in a mechanical testing system using a $50.8 \mathrm{~mm}$ diameter indenter at a constant displacement rate of $1.27 \mathrm{~mm} / \mathrm{min}$. The displacement and load were monitored during the indentation test and the composite was unloaded when the data showed a sudden drop in load (due to damage growth), as shown in Figure 4Figure 4b.

Figure 5 Figure $5 \mathrm{a}$ gives a photo of the CFRP plate after the quasi-static indentation test. Figure 5 Figure $5 \mathrm{~b}$ is a zoomed in view which shows a very slight indentation on the surface with no other surface damage. Following the quasistatic indentation test, an ultrasonic Cscan was used to quantify the resulting damage under the plate surface. The Cscan data were collected in an immersion tank using a $10 \mathrm{MHz}$ transducer and a scan step size of $0.25 \mathrm{~mm}$. The Cscan image in Figure 5Figure $5 \mathrm{c}$ shows multiple delaminations within the $32.3 \mathrm{~mm} \times 22.6 \mathrm{~mm}$ damage zone, occurring at various depths through the plate thickness.

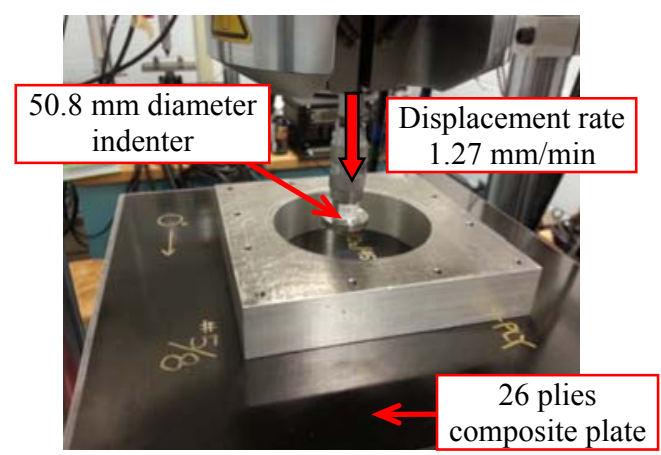

(a)

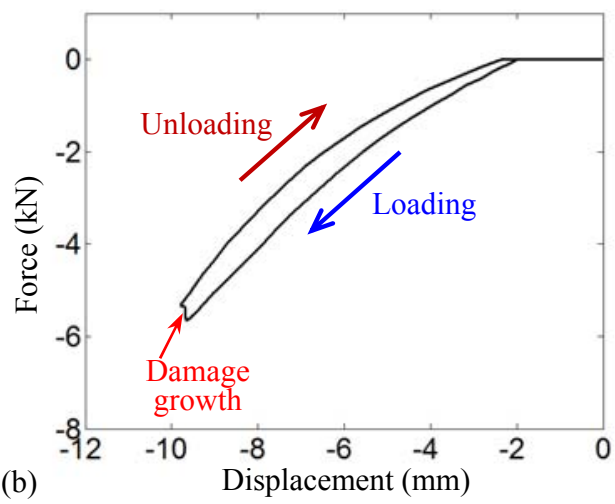

(b)

Figure 4. Quasi-static indentation for delamination damage growth: (a) the indentation setup; (b) the curve of indentation force versus displacement. 


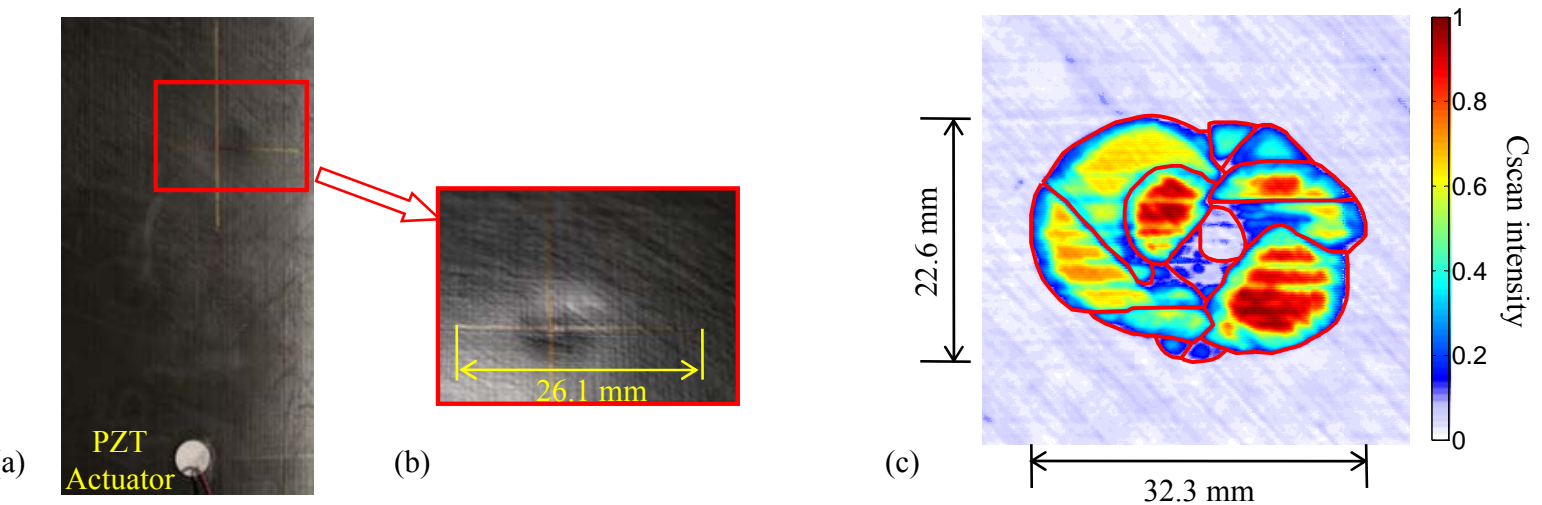

Figure 5. The 26 ply laminate after the quasi-static indentation: (a) the specimen with PZT actuator, (b) a close look of the indentation caused by the impact, and (c) a C-scan image showing the internal delamination damage generated by the indentation test. The red solid lines in figure (c) represents the boundaries extracted from the Cscan image.

\subsection{Delamination detection and quantification}

To perform the spatial wavenumber imaging, a $7 \mathrm{~mm}$ round PZT was installed on the plate surface (shown in Figure 5Figure 5a). The PZT is used to generate guided waves. Two excitation frequencies are studied, $100 \mathrm{kHz}$ and 500 $\mathrm{kHz}$. The wavefields were measured by using the SLDV. Figures $6 \mathrm{a}$ and b give wavefield snapshots for the $100 \mathrm{kHz}$ excitation at $45 \mu s$ for the pristine and damaged cases respectively. The pristine wavefield shows a single incident wave mode with the wavelength of $11.5 \mathrm{~mm}$. The damaged wavefield shows strong wave interactions at the damage region, compared to the pristine case. As shown in the figures, the low excitation frequency leads to a long wavelength mode interacting with the damage. Figures $7 \mathrm{a}$ and $\mathrm{b}$ show the wavefield at $40 \mu \mathrm{s}$ after the $500 \mathrm{kHz}$ excitation. Significantly different scattering behavior is observed for the shorter wavelength mode (compared to $100 \mathrm{kHz}$ ).

The spatial wavenumber imaging method is applied to the guided wavefield, for the purpose of detecting and evaluating the impact-induced delamination damage. Figure 8a plots the spatial wavenumber imaging result over the test area at $100 \mathrm{kHz}$. Figure $8 \mathrm{~b}$ shows the wavenumber result for $500 \mathrm{kHz}$. Both imaging results show an area with larger wavenumbers that is approximately $30 \mathrm{~mm} \times 20 \mathrm{~mm}$. The high frequency result highlights delamination regions that are less apparent in the $100 \mathrm{kHz}$ result (for example, the red region in the upper left of the damage in figure $8 \mathrm{~b}$ ). The results demonstrate that frequency does have an effect on the resulting wavenumber image, and thus affects the resulting quantification. Compared to the Cscan result, the detected damage agrees fairly well the Cscan result, however regions of the damage do not readily show up in the wavenumber image. The detected shape in the wavenumber image does roughly resemble the shape of the Cscan result that is plotted as red solid lines in Figure 8. The detectability of portions of the damage is expected to be dependent on both depth and frequency. Prior work showed detectability with wavenumber imaging is affected by those factors, and the results presented here re-confirm the dependency [19]. Further work is needed to assess the accuracy of quantification and the dependence on excitation frequency.
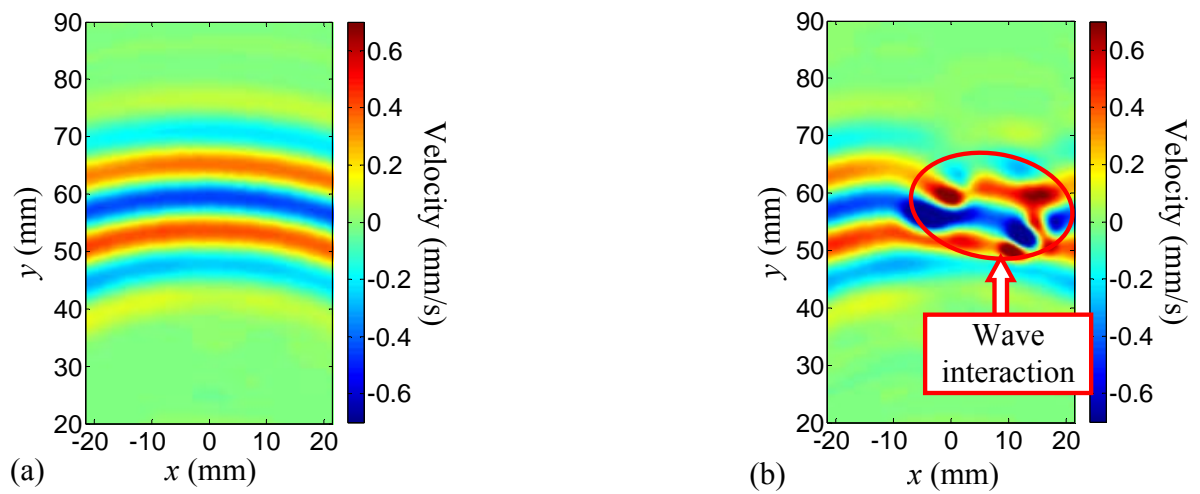

Figure 6. SLDV wavefield images at $45 \mu$ s for $100 \mathrm{kHz}$ excitation: (a) for the pristine plate, and (b) for the plate with damage. 

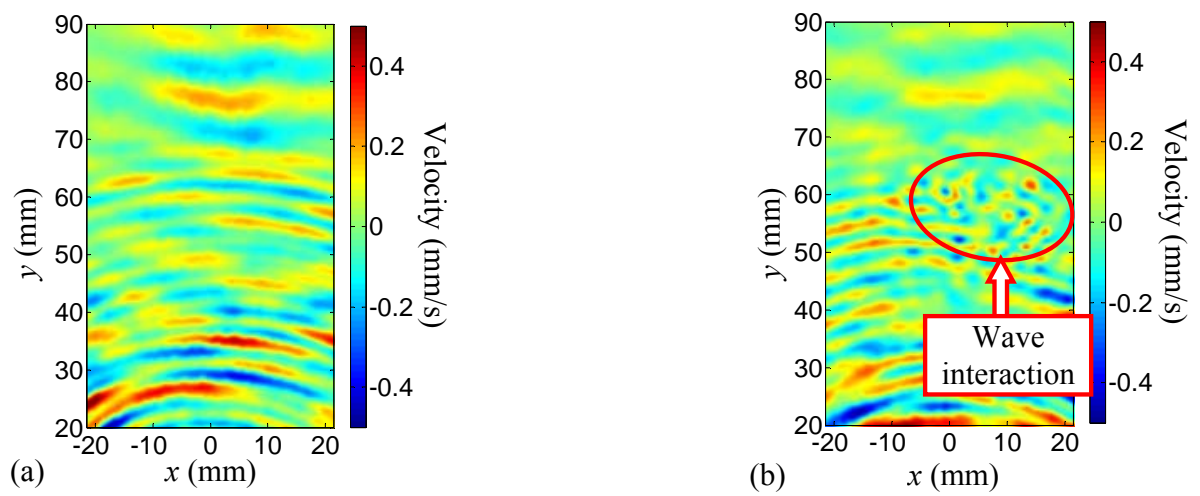

Figure 7.

SLDV wavefield images at $40 \mu$ s for $500 \mathrm{kHz}$ excitation:

(b)

$$
x(\mathrm{~mm})
$$

damage.
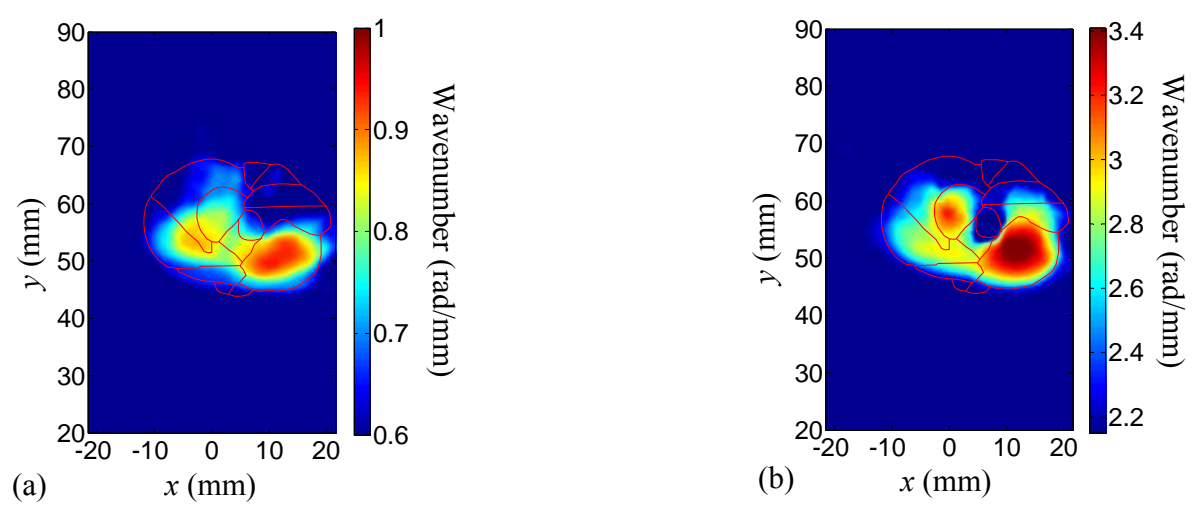

Figure 8. Spatial wavenumber imaging results: (a) $100 \mathrm{kHz}$, (b) $500 \mathrm{kHz}$.

\section{CONCLUSIONS}

This paper studies impact induced delamination detection and quantification by using guided wavefield data and spatial wavenumber imaging. The complex geometry impact-like delamination is created through a quasi-static indentation on a CFRP plate. To detect and quantify the impact delamination in the CFRP plate, PZT-SLDV sensing and spatial wavenumber imaging are performed. In the PZT-SLDV sensing, the guided waves are generated from the PZT, and the high spatial resolution guided wavefields are measured by the SLDV. The guided wavefield data acquired from the PZT-SLDV sensing represent guided wave propagation in the composite laminate and include guided wave interaction with the delamination damage. The measured guided wavefields are analyzed through the spatial wavenumber imaging method, which generates an image containing the dominant local wavenumber at each spatial location. The spatial wavenumber imaging result for the simple single layer Teflon insert delamination provided quantitative information on delamination damage size and location. The location of delamination damage is indicated by the area with larger wavenumbers in the spatial wavenumber image. The impact-like delamination results only partially agreed with the damage size and shape. The results also demonstrated the dependence on excitation frequency. Future work will further investigate the accuracy of the wavenumber imaging method for real composite damage and the dependence on frequency of excitation. 


\section{ACKNOWLEDGEMENT}

The authors would like to thank (1) the non-reimbursement space act umbrella agreement SAA1-1181 between South Carolina Research Foundation (SCRF) and the National Aeronautics and Space Administration (NASA) Langley Research Center, and (2) SC NASA EPSCoR Research and Education Awards Program (REAP) 21-NE-USC_Yu-RGP.

\section{REFERENCES}

1. Ip, K. H., and Mai, Y. W., "Delamination Detection in Smart Composite Beams Using Lamb Waves," Smart Mater Struct, 13, 544-551 (2004).

2. Staszewski, W. J., Mahzan, S., and Traynor, R., "Health Monitoring of Aerospace Composite Structures-Active and Passive Approach," Compos Sci Technol, 69, 1678-1685 (2009).

3. Giurgiutiu, V., and Soutis, C., "Enhanced Composites Integrity through Structural Health Monitoring," Appl Compos Mater, 19, 813-829 (2012).

4. Prasad, S. M., Balasubramaniam, K., and Krishnamurthy, C. V., "Structural Health Monitoring of Composite Structures Using Lamb Wave Tomography," Smart Mater Struct, 13, N73-N79 (2004).

5. Wang, L., and Yuan, F. G., "Group Velocity and Characteristic Wave Curves of Lamb Waves in Composites: Modeling and Experiments," Compos Sci Technol, 67, 1370-1384 (2007).

6. Kundu, T., Das, S., Martin, S. A., and Jata, K. V., "Locating Point of Impact in Anisotropic Fiber Reinforced Composite Plates," Ultrasonics, 48, 193-201 (2008).

7. Su, Z. Q., Cheng, L., Wang, X. M., Yu, L., and Zhou, C., "Predicting Delamination of Composite Laminates Using an Imaging Approach," Smart Mater Struct, 18, 074002 (2009).

8. Castaings, M., and Hosten, B., "Ultrasonic Guided Waves for Health Monitoring of High-Pressure Composite Tanks," Ndt\&E Int, 41, 648-655 (2008).

9. Salamone, S., Bartoli, I., di Scalea, F. L., and Coccia, S., "Guided-Wave Health Monitoring of Aircraft Composite Panels under Changing Temperature," J Intel Mat Syst Str, 20, 1079-1090 (2009).

10. Salas, K. I., and Cesnik, C. E. S., "Guided Wave Structural Health Monitoring Using Clover Transducers in Composite Materials," Smart Mater Struct, 19, 015014 (2010).

11. Song, F., Huang, G. L., and Hu, G. K., "Online Guided Wave-Based Debonding Detection in Honeycomb Sandwich Structures," Aiaa J, 50, 284-293 (2012).

12. Leckey, C. A. C., Rogge, M. D., and Parker, R., "Guided Waves in Anisotropic and Quasi-Isotropic Aerospace Composites: Three-Dimensional Simulation and Experiment," Ultrasonics, 54, 385-394 (2014).

13. Guo, N., and Cawley, P., "The Interaction of Lamb Waves with Delaminations in Composite Laminates," Journal of Acoustic Society of America, 94, 2240-2246 (1993).

14. Petculescu, G., Krishnaswamy, S., and Achenbach, J. D., "Group Delay Measurements Using Modally Selective Lamb Wave Transducers for Detection and Sizing of Delaminations in Composites," Smart Mater Struct, 17, 015007 (2008).

15. Purekar, A. S., and Pines, D. J., "Damage Detection in Thin Composite Laminates Using Piezoelectric Phased Sensor Arrays and Guided Lamb Wave Interrogation," J Intel Mat Syst Str, 21, 995-1010 (2010).

16. Michaels, T. E., Michaels, J. E., and Ruzzene, M., "Frequency-Wavenumber Domain Analysis of Guided Wavefields," Ultrasonics, 51, 452-466 (2011).

17. Yeum, C. M., Sohn, H., Ihn, J. B., and Lim, H. J., "Instantaneous Delamination Detection in a Composite Plate Using a Dual Piezoelectric Transducer Network," Compos Struct, 94, 3490-3499 (2012).

18. Sohn, H., Dutta, D., Yang, J. Y., DeSimio, M., Olson, S., and Swenson, E., "Automated Detection of Delamination and Disbond from Wavefield Images Obtained Using a Scanning Laser Vibrometer," Smart Mater Struct, 20, 045017 (2011).

19. Rogge, M. D., and Leckey, C. A. C., "Characterization of Impact Damage in Composite Laminates Using Guided Wavefield Imaging and Local Wavenumber Domain Analysis," Ultrasonics, 53, 1217-1226 (2013).

20. Flynn, E. B., Chong, S. Y., Jarmer, G. J., and Lee, J. R., "Structural Imaging through Local Wavenumber Estimation of Guided Waves," Ndt\&E Int, 59, 1-10 (2013).

21. Mesnil, O., Leckey, C. A. C., and Ruzzene, M., "Instantaneous and Local Wavenumber Estimations for Damage Quantification in Composites," Structural Health Monitoring(2014). 
22. Hayashi, T., and Kawashima, K., "Multiple Reflections of Lamb Waves at a Delamination," Ultrasonics, 40, 193197 (2002).

23. Ramadas, C., Balasubramaniam, K., Joshi, M., and Krishnamurthy, C. V., "Interaction of the Primary AntiSymmetric Lamb Mode (A0) with Symmetric Delaminations: Numerical and Experimental Studies," Smart Mater Struct, 18, 085011 (2009).

24. Sohn, H., Dutta, D., Yang, J. Y., Park, H. J., DeSimio, M. P., Olson, S. E., and Swenson, E. D., "Delamination Detection in Composites through Guided Wave Field Image Processing," Compos Sci Technol, 71, 1250-1256 (2011).

25. Glushkov, E., Glushakova, N., Golub, M. V., Moll, J., and Fritzen, C. P., "Wave Energy Trapping and Localization in a Plate with a Delamination," Smart Mater Struct, 21, 125001 (2012).

26. Tian, Z., Yu, L., and Leckey, C. A. C., "Delamination Detection and Quantification on Laminated Composite Structures with Lamb Waves and Wavenumber Analysis," J Intel Mat Syst Str(2014).

27. Darnton, A., and Ruzzene, M., "Damage Mapping in Composites with Phase Gradient," ASME 2014 Conference on Smart Materials, Adaptive Structures and Intelligent Systems, Newport, Rhode Island, USA. 1, SMASIS2014-7685.

28. Staszewski, W. J., Mahzan, S., and Traynor, R., "Health Monitoring of Aerospace Composite Structures - Active and Passive Approach," Compos Sci Technol, 69, 1678-1685 (2009).

29. Alleyne, D. N., and Cawley, P., "A Two Dimensional Fourier Transform Method for the Measurement of Propagating Multimode Signals," Journal of Acoustic Society of America, 89, 1159-1168 (1991).

30. Hayashi, T., and Kawashima, K., "Single Mode Extraction from Multiple Modes of Lamb Wave and Its Application to Defect Detection," JSME International Journal Series A, Solid Mechanics and Material Engineering, 46, 620-626 (2003).

31. Ruzzene, M., "Frequency-Wavenumber Domain Filtering for Improved Damage Visualization," Smart Materials and Structures, 16, 2116-2129 (2007).

32. Tian, Z., Leckey, C. A. C., Seebo, J. P., and Yu, L., "Guided Wave Delamination Detection and Quantification with Wavefield Data Analysis" New York: ASME, Newport, Rhode Island(2014).

33. Williams, G., Trask, R., and Bond, I., "A Self-Healing Carbon Fibre Reinforced Polymer for Aerospace Applications," Compos Part a-Appl S, 38, 1525-1632 (2007). 